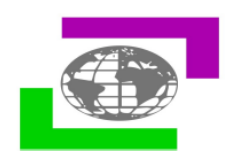

\title{
Process Validation in
}

\section{Pharmaceutical Product Development: An Overview \\ Sreenivasa.G.M*, Sriranga.T, Naveen Banakar, Vaishakha Kori, ChetankumarVasanad, Pavankumar.S.P}

P.G. Dept. Of Quality Assurance, S.C.S College of Pharmacy, Harapanahalli, Karnataka, India.

Article Info: Received 09 September 2021; Accepted 08 October 2021

DOI: https://doi.org/10.32553/jbpr.v10i5.883

Corresponding author: Dr. Sreenivasa G.M

Conflict of interest statement: No conflict of interest

\begin{abstract}
As per GMP process validation is very important and required part in product development. Process Validation is required for ensuring and providing the documentary evidence and also required for giving the Surety that process by which the formulation is prepared is capable of consistently producing the product of required quality and purity.

A validated manufacturing process is one which has been proved to do what it purports on is represented to do. The proof of validation is obtained through the collection and evaluation of data, preferably, beginning from the process development phase and Continuing through the production phase. Validation necessarily includes process qualification (the qualification of materials, equipment Systems, buildings, personnel), but it also includes the control on the entire process for repeated batches or runs. This article gives a general introduction and overview about process validation in pharmaceutical manufacturing process and its importance.
\end{abstract}

Keywords: GMP, Validation, validation manufacturing process.

\section{Introduction}

The word validation simply means assessment of validity or action of proving effectiveness. Validation is a team effort where it involves people from various disciplines of the plant. This principle incorporates the understanding that the following conditions exist: Quality, safety, and efficacy are designed or built into the product. Quality cannot be adequately assured merely by in-process and finished product inspection or testing each step of a manufacturing process is controlled to assure that the finished product meets all quality attributes including specifications ${ }^{1}$.
Validation is a concept that has been evolving continuously since its first formal appearance in the United States in 1978. The concept of validation has expanded through the years to encompass a wide range of activities from analytical methods used for the quality control of the drug substances and drug products to computerized systems for clinical trials ${ }^{2}$.

FDA, or any other food and drugs regulatory agency around the globe not only ask for a product that meets its specification but also require a process, procedures, intermediate stages of inspections, and testing adopted during 
manufacturing are designed such that when they are adopted they produce consistently similar, reproducible, desired results which meet the quality standard of product being manufactured and complies the Regulatory and Security Aspects. Such procedures are developed through the process of validation. This is to maintain and assure a higher degree of quality of food and drug products. Validation is defined as Establishing documented evidence which provides a high degree of assurance that a specific process will consistently produce a product meeting its pre-determined specifications and quality attributes ${ }^{3}$.

\section{Why Validation is Required ${ }^{3}$ ?}

* Validation requires an appropriate and sufficient infrastructure including: organization, documentation, personnel and finances.

* Involvement of management and quality assurance personnel.

* Personnel with appropriate qualifications and experience.

* Extensive preparation and planning before validation is performed.

* Validation should be performed: - for new premises, equipment, utilities and systems, and processes and procedures: -at periodic intervals; and when major changes have been made.

* Validation in accordance with written protocols.

* Validation over a period, e.g. at least three consecutive batches (full production scale) to demonstrate consistency. (Worst case situations should be considered.)

* Significant changes (facilities, equipment, processes)-should be validated.

* Risk assessment approach used to determine the scope and extent of validation needed.

\section{History of Validation}

The concept of validation was first proposed by two FDA officials, Ted Byers and Bud Loftus, in the mid 1970's in order to improve the quality of pharmaceuticals (Agalloco 1995). It was proposed in direct response to several problems in the sterility of large volume parental market. The first validation activities were focused on the processes involved in making these products, but quickly spread to associated process of pharmaceutical.

U.S.F.D.A. was the pioneer in advocating the concept of process validation, but till 29th September 1978 the definition of process validation did not appear in any part of literature of U.S.F.D.A. no cGMP regulations talked anything about process validation ${ }^{4}$.

\section{Definitions $^{5-7}$}

\section{European commission}

1991 -Validation-“Act of proving, in accordance of GMPs that Any..." process actually leads to expected results.

2000 -"Documented evidence that the process, operated within established Parameters, can perform effectively and reproducibly to produce a Medicinal product meeting its predetermined specifications and quality attributes".

\section{US FDA Definition}

"Process validation is establishing documented evidence which provides a high degree of assurance that a specified process will consistently produce a product meeting its predetermined specifications and quality characteristics."

\section{ICH Definition}

"Process Validation is the means of ensuring and providing documentary evidence that processes within their specified design parameters are capable of repeatedly and reliably producing a finished product of the required quality."

\section{WHO Definition}

"The documented act of proving that any procedure, process, equipment, material, activity or system actually leads to expected result".

\section{Benefits of Validation}

\section{Reduction of quality cost}


Through proper validation, the cost of the following process can be optimized.

a. Preventive costs are costs incurred in order to prevent failure and reduce appraisal costs.

b. Appraisal costs of inspection, testing and quality evaluation.

c. Internal failure costs.

d. External failure costs that associated with a nonconformance condition after the product had left the company's ownership.

\section{Process optimization}

The optimization of the facility, equipment system and closures etc. results in a product that meets quality requirements at the lower costs. Trained, qualified people are the key elements in process optimization that results in improving efficiency and productivity.

\section{Assurance of quality}

Validation and process control are the heart of GMPs. Without validated and controlled process it is impossible to achieve quality products. Hence validation is a key element in assuring the quality of the product.

\section{Safety}

Validation can also result in increased operator safety. Properly calibrated, validated instruments and gauges used to reduce accidents and results in safety.

\section{Better customer quality}

Through proper validation, market recall is avoided which results in better customer care and quality of the product ${ }^{8}$.

\section{Advantages of Validation}

1. Enhanced reporting capability.

2. Improved ability to set target parameters and control limits for routine production, correlating with validation results.

3. Enhanced data and evaluation capabilities and increased confidence about process reproducibility and product quality.

4. Enhanced ability to statistically evaluate process performance and product variables e.g., individuals, mean, range, control limits ${ }^{9}$.

\section{Types of Validation}

Validation is classified into following types:

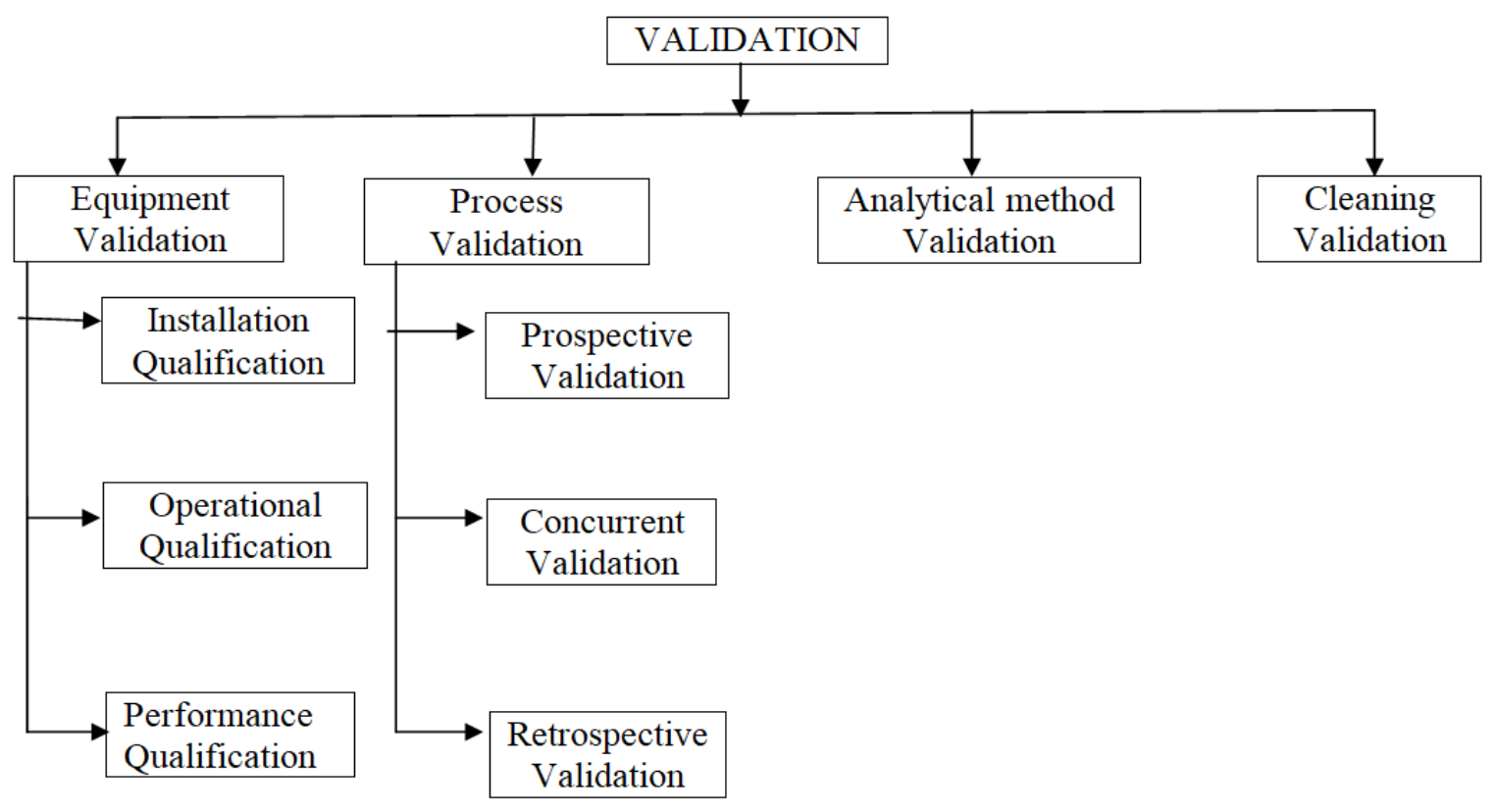

Fig. 1: Validation types 


\section{Equipment Validation}

The key concept of validation is to give a high degree of reported $\neg$ confirmation that the equipment and the procedure conform to the written guidelines. The degree (or intensity) is dictated by the complexity of the device or system. The validation should give the essential data and test methods required to give that the device and technique meet determined prerequisites ${ }^{11}$.

\section{Analytical Method Validation}

Validation of an analytical approach is established through laboratory research, that the execution attributes of the procedure meet the requirements for the proposed scientific application. Validation is required for any new or altered procedure to verify that it is fit for giving predictable and dependable outcomes, once used by various administrators by usage of comparable instrumentation inside the similar or absolutely distinct laboratories ${ }^{12}$.

Method validation is a reported program that offers with that the processing system will give a high level of affirmation to meet its predicated acceptance basis.

\section{Cleaning Validation}

Cleaning validation is a reported proof with a high level of confirmation that can uniformly clean a system or equipment to already determined and specification criteria. Cleaning approval is a reported procedure that demonstrates the efficacy and consistency in cleaning pharmaceutical production equipment. The goal of cleaning approval is to check the viability of the cleaning system for the expulsion of product deposits, degradants, additives, excipients, or cleaning agents and in a the control of potential microbial contamination ${ }^{13-}$ 14

\section{Process Validation}

"Process Validation is establishing documented evidence which provides a high degree of assurance that a specific process will consistently produce a product meeting its pre- determined specifications and quality characteristics."

It is beneficial to the manufacturer in many ways $^{15}$

- It deepens the understanding of processes, decreases the risk, preventing problems and thus assures the smooth running of the process.

* It decreases the risk of defect costs.

* It decreases the risk of regulatory noncompliance.

* A fully validated process may require less in-process controls and end- product testing.

Validation should thus be considered in the following situations:

Totally new process.

* New equipment.

* Process and equipment which have been altered to suit changing priorities.

* Process where the end-product test is poor and an unreliable indicator of product quality.

\section{Basic Concept of Process Validation}

* Calibration, verification and maintenance of process equipment.

* Requalification or revalidation.

* Establishing specifications and performance characteristics.

* Election of methods, process and equipment to ensure the product meets specifications.

- Qualification or validation of process and equipment.

* Testing the final product, using validated analytical methods, in order to meet specifications.

* Challenging, auditing, monitoring or sampling the recognized critical key steps of the process.

\section{Objectives of process validation}

* The manufacturing process, in addition to the individual equipment, must be validated.

* The goal is to create a robust manufacturing process that consistently produces a drug product with minimal variation that adheres 
to quality criteria of purity, identity, and potency.

* A validation plan for the manufacturing process should be drafted and executed by engineers in order to satisfy guidelines. The validation plan usually involves just a PQ section.

* Just as equipment validation, major changes after the initial validation will result in the need for subsequent revalidation.

* In the end, process validation will ensure a robust product that is highly reproducible over time.

\section{Advantages of process validation}

* Expanded real time monitoring and adjustment of process.

* Enhanced ability to statistically evaluate process performance and product variables. e.g., individuals; mean; range; control limits.

* Enhanced data and evaluation capabilities and increased confidence about process reproducibility and product quality.

* Improved ability to set target parameters and control limits for routine production, correlating with validation results.

* Enhanced reporting capability.

\section{TYPES OF PROCESS VALIDATION}

\section{Prospective Validation}

a. Prospective validation is usually undertaken whenever a new formula, process and/or facility need to be validated before routine pharmaceutical production starts. It is also usually employed when sufficient historical data is either unavailable or insufficient and in process and final product testing is inadequate to ensure high degree of confidence for product quality characteristics and reproducibility ${ }^{16}$.

b. In prospective validation, the validation protocol is executed before the process is put into commercial use. During the product development phase, production process should be categorized into individual steps. Each step should be evaluated on the basis of experience or theoretical consideration to determine the critical parameters that may affect the quality of the finished product.

c. Each experiment should be planned and documented fully in an authorized protocol. All equipments, production environment and the analytical testing methods to be used should have been fully validated.

d. Master batch documents can be prepared only after the critical parameters of the process have been identified and machine setting, component specification and environmental condition have been determined.

e. Using this defined process a series of batches should be produced. In theory, the number of process runs carried out and observations made should be sufficient to allow the normal extent of variation and trends to be established to provide sufficient data for evaluation.

f. It is generally considered acceptable that three consecutive batches/runs with in the finally agreed parameters, giving product of the desired quality would constitute a proper validation of the process.

g. During the processing of the validation batches extensive sampling and testing should be prepared on the product at various stages and should be documented comprehensively. Detailed testing should also be done on the final product in its package.

h. Upon completion of the review, recommendations should be made on the extent of monitoring and the in- process controls necessary for routine production. These should be incorporated into the batch manufacturing and packaging record or into appropriate SOP. Limits frequencies and actions to be taken in the event of the limits being exceeded should be specified ${ }^{17}$.

\section{Concurrent Validation}

It is similar to the prospective, except the operating firm will sell the product during the qualification runs to the public at its market price. This validation involves in process monitoring of critical processing steps and 
product testing. This helps to generate and documented evidence to show that the production process is in state of control ${ }^{18}$.

Concurrent validation is appropriate when

a. It is not possible to complete a validation program before routine manufacturing starts and it is known in advance that finished product will be for sale. E.g. During transference of process to contract manufacturer;

b. It is more appropriate to validate process during routine production due to well understanding of process. E.g. On change in tablet shape or strength;

c. Extensive testing and monitoring ensures the desired quality characteristics of product with high degree of confidence.

\section{Retrospective Validation}

Retrospective validation is the validation of a process based on accumulated historical production, testing, control and other information for a product already in production and distribution. This type of validation make use of historical data and information which may be found in batch record, production log books, lot records, controls charts, test and inspection results, customer complaints or lack of complaints field, failure report, service report and audit report ${ }^{19}$.

Further, large historical data set available may provide higher confidence and better picture than data generated from few trials runs in prospective validation. This type of validation is acceptable only for well established processes in which critical quality attributes and critical process parameters are identified and documented. Besides that appropriate in process specification and control should be identified and documented; And there should not be excessive process/product failure other than operator error or equipment failure unrelated to equipment suitability.

The number of batches to review will depend on the process, but in general, data from 10 to 30 consecutive batches should be examined to assess process consistency. The review should include any batches that failed to meet specification. However, any discrepancies or failure in the historical data may be excluded provided there is sufficient evidence that the failure was caused by isolated occurrences.

E.g. employee error, and were not result of process variations.

\section{Revalidation}

1. Revalidation is the repetition of the validation process or a specific part of it. It is either performed periodically to ascertain the process or to incorporate changes in the procedure $^{16}$.

2. Documentation requirements will be the same as for the initial validation of the process. Revalidation becomes necessary in certain situations.

a. Changes in raw materials (Physical properties such as density, viscosity, particle size distribution and moisture etc., that may affect the process or product).

b. Changes in the source of active raw material manufacturer.

c. Changes in packaging material (Primary container/ closure system).

d. Changes in the process (e.g. mixing time, drying temperature and batch size).

e. Changes in the plant/ facility ${ }^{20}$.

\section{Phases in Process Validation}

The activities relating to validation studies may be classified into three phases.

\section{Phase:1 Pre validation phase or the qualification phase}

It covers all activities relating to product research and development, formulations, pilot batch studies, scale up studies, transfer of technology to commercial scale batches, establishing stability conditions, storage and handling of in- process and finished dosage forms, equipment qualification, Installation qualification, Master production documents, operational qualification, process capability ${ }^{21}$

Phase:2 Process validation phase 
This phase is designed to verify that all established limits of the critical process parameters are valid and that satisfactory products can be produced even under the worst case condition. It represents the actual studies or trials conducted to show.

1. That all systems subsystem or unit operations of a manufacturing process perform as intended.

2. That all critical parameters operate within their assigned control limit.

3. That such studies and trials, which form the basis of process capability design and testing, are verifiable and certifiable through proper documentation.

\section{Phase:3 Validation Maintenance phase}

This phase requires frequent review of all process related documents, including validation audit report to assure that there have been no changes, deviations, failures, modifications to the production process and that all SOP have been followed, including change control procedure. At this stage the validation team also assures that there have been no changes, deviations that should have resulted in requalification and revalidation.

\section{Validation Protocol}

A written plan stating how validation will be conducted, including test parameters, product characteristics, production and packaging equipment and decision points on what constitutes acceptable test results. This document should give details of critical steps of the manufacturing process that should be measured, the allowable range of variability and the manner in which the system will be tested.

The validation protocol provides a synopsis of what is hoped to be accomplished. The protocol should list the selected process and control parameters, state the number of batches to be included in the study and specify how the data, once assembled, will be treated for relevance. The date of approval by the validation team should also be noted.
In the case where a protocol is altered or modified after its approval, appropriate reasoning for such a change must be documented ${ }^{22}$.

The validation protocol should be numbered, signed and dated, should contain as a minimum the following information:

1. Objectives, scope of coverage of the validation study.

2. Validation team membership, their qualifications and responsibilities.

3. Type of validation: prospective, concurrent, retrospective, revalidation.

4. Number and selection of batches to be on the validation study.

5. A list of all equipment to be used; their normal and worst case operating parameters.

6. Outcome of IQ, OQ for critical equipment.

7. Requirements for calibration of all measuring devices.

8. Critical process parameters and their respective tolerances.

9. Description of the processing steps: copy of the master documents for the product.

10. Sampling points, stages of sampling, methods of sampling and sampling plans.

11. Statistical tools to be used in the analysis of data.

12. Training requirements for the processing operators.

13. Validated test methods to be used in inprocess testing and for the finished product.

14. Specifications for raw and packaging materials and test methods.

15. Forms and charts to be used for documenting results.

16. Format for presentation of results, documenting conclusions and for approval of study results ${ }^{23-26}$. 


\section{Validation Master Plan ${ }^{27-28}$}

The validation master plan should provide an overview of the entire validation operation, its organizational structure, its content and planning. The main elements of it being the list/inventory of the items to be validated and the planning schedule. All validation activities relating to critical technical operations, relevant to product and process controls within a firm should be included in the validation master plan. It should comprise all prospective, concurrent and retrospective validations as well as revalidation.

The Validation Master Plan should be a summary document and should therefore be brief, concise and clear. It should not repeat information documented elsewhere but should refer to existing documents such as policy documents, SOP's and validation protocols and reports.

The format and content should include:

* Introduction: validation policy, scope, location and schedule.

* Organizational structure: personnel responsibilities.

* Plant/process/product description: rational for inclusions or exclusions and extent of validation.

* Specific process considerations that are critical and those requiring extra attention.

* List of products/ processes/ systems to be validated, summarized in a matrix format, validation approach.

* Re-validation activities, actual status and future planning.

* Key acceptance criteria.

* Documentation format.

* Reference to the required SOP's.

* Time plans of each validation project and sub-project.

\section{Validation Parameters}

The main aim of method validation is to produce proof that the method will what it is supposed to do, accurately, reliable and consistent. The validation parameters as per ICH guidelines are described below:

\section{Accuracy}

Accuracy is expressed as the nearness of agreement $\neg$ between the values found and values that are already available. It can also be defined as the closeness between the true value and the observed value. It is sometimes called as trueness, and it could be determined by using at least 9 determinations over a minimum of 3 concentrations over the specified range ${ }^{29}$.

Accuracy of prochlorperazine maleate (PRO) and betahistine hydrochloride (BET) was studied by the standard addition method at three different levels $(50 \%, 100 \%$, and 120\%). A known amount of drug was added to the preanalyzed sample and percentage recovery calculated. When this method was used for accuracy, the recovery was found to be $99.38 \%$ for betahistine hydrochloride and $99.11 \%$ for prochlorperazine maleate ${ }^{30}$.

For the concurrent determination of nitazoxanide and ofloxacin accuracy was studied by the standard addition method at five different levels $(50 \%, 75 \%, 100 \%, 125 \%$, and $150 \%$ ). The results indicate that the recoveries were observed to be in the range of $80 \%$ to $120 \%$, therefore, the method is accurate.

For the concurrent determination of nitazoxanide and ofloxacin accuracy was studied by the standard addition method at five different levels $(50 \%, 75 \%, 100 \%, 125 \%$, and $150 \%$ ). The results indicate that the recoveries were observed to be in the range of $80 \%$ to $120 \%$, therefore, the method is accurate ${ }^{31}$.

\section{Precision}

The exactness of an analytical procedure expresses the nearness of agreement (degree of scatter) between a group of measurements obtained from different sampling of a uniform sample underneath the prescribed conditions ${ }^{32}$. Precision may be taken into consideration at 3 levels:

Repeatability: It expresses the exactness below a similar operating condition over a brief interval of time and also referred as intra-assay precision. A minimum of six 
replicates test preparation of a similar or consistent sample ready at the $100 \%$ check $^{33}$.

* Intermediate precision: It expresses the exactness under inside research laboratories, in distinct days, through distinct analyst, on distinct instruments/equipment. Two different analysts each preparing six sample solutions, as per specified method ${ }^{34}$.

* Reproducibility: It refers to the precision between different analytical labs. Every research facility set up an aggregate of six sample solutions, according to the analytical technique.

The precision of prochlorperazine maleate (PRO) and betahistine hydrochloride (BET) method was determined by inter-day and intraday variation ( $\% \mathrm{RSD})$. Intra-day precision was performed by analyzing standard drug solutions within the calibration range, three times on the same day. Inter-day precision was performed by analyzing drug solutions within the calibration range on three different days over a period of seven days. The low $\%$ RSD values of inter-day (1.02 to $1.48 \%$ for BET at $252.9 \mathrm{~nm}$ and 0.67 to $0.82 \%$ for PRO at $260.15 \mathrm{~nm}$ ) and intraday ( 0.77 to $1.09 \%$ for BET at $252.9 \mathrm{~nm}$ and 0.27 to $0.61 \%$ for PRO at $260.15 \mathrm{~nm}$ variation for BET and PRO, revealed that the method is precise.

For simultaneous estimation of nitazoxanide and ofloxacin precision performed by injecting six replicates of a sample prepared from commercial tablets and assay was calculated to determine the repeatability of retention time and a peak area of standard and samples. The percentage relative standard deviation (\% RSD) values for the area of nitazoxanide and ofloxacin were 0.44 and $0.2 \%$ and RSD values for a retention time of nitazoxanide and ofloxacin were $0.44 \%$ for both the drugs.

The precision of paracetamol was checked by injecting a solution of $80 \mu \mathrm{g} / \mathrm{ml}$ for six times in same days, different days, and in a different time interval on the same day. The $\%$ RSD was found to be less than $3 \%$, which showed good precision $^{35}$.

\section{Specificity}

For every stage of development, the analytical $\neg$ technique should demonstrate specificity. The technique was should have the power to unequivocally assess the analyte of interest whereas within the presence of all expected parts, which can encompass degradants, excipients/sample matrix, and sample blank peaks $^{36}$.

Specificity was performed to determine the retention time of each drug in a mixture and in the sample. The retention time of standard drugs individually was determined, and it was found to be $3.750 \mathrm{~min}$ and $1.533 \mathrm{~min}$ for nitazoxanide and ofloxacin and retention time of both drugs in the standard mix was found to be $3.760 \mathrm{~min}$ for nitazoxanide and $1.542 \mathrm{~min}$ for ofloxacin respectively.

\section{Limit of detection (LOD)}

Lowest quantity of an analyte which $\neg$ may be detected by the chromatographical separation however it is not necessary that this quantity will quantify as a precise value. A blank resolution is injected and peak to peak quantitative noise relation we have to calculate from blank chromatograms. Then, calculate the concentration at the signal to quantitative noise relation is concerning $3: 1$.

LOD can be expressed as

$\mathrm{LOD}=3.3 \mathrm{SD} / \mathrm{S}$ Where,

$\mathrm{SD}=$ Standard deviation of response,

$\mathrm{S}=$ Slope of calibration curve ${ }^{37}$.

The LOD value of betahistine hydrochloride (BET) and prochlorperazine maleate (PRO) is $0.29 \mu \mathrm{g} / \mathrm{ml}$ and $0.34 \mu \mathrm{g} / \mathrm{ml}$ respectively.

LOD of the paracetamol was studied by the signal to noise ratio, and the result was found to be $120 \mu \mathrm{g} / \mathrm{ml}$.

Limit of Quantitation (LOQ): It is characterized by the least $\neg$ quantity of an analyte that can be quantified with exactness and precision.

LOQ can be communicated as 
$\mathrm{LOQ}=10 \mathrm{SD} / \mathrm{S}$

Where SD $=$ Standard deviation of response, $\mathrm{S}=$ Slope of calibration curve $\mathrm{e}^{38}$.

The LOQ value of betahistine hydrochloride (BET) and prochlorperazine maleate $(\mathrm{PRO})$ is $0.957 \mu \mathrm{g} / \mathrm{ml}$ and $1.12 \mu \mathrm{g} / \mathrm{ml}$ respectively.

LOQ of the paracetamol was studied by the signal to noise ratio, and the result was found to be $360 \mu \mathrm{g} / \mathrm{ml}^{35}$.

Some usual techniques, methods for the assessment of LOD and LOQ are as follows:

Visual inspection,

- Signal to noise ratio,

* Standard deviation of the blank, and

* Regression line at low concentrations ${ }^{39}$.

\section{Linearity}

Linearity may be characterized as the capacity of an $\neg$ analytical technique to produce outcomes which are directly related to the concentration of an analyte in the ${ }^{40}$.

Standard solution of betahistine hydrochloride (BET) and prochlorperazine maleate (PRO) was taken in a $10 \mathrm{ml}$ volumetric flask and diluted with $0.1 \mathrm{~N} \mathrm{HCL}$ to get the final concentration in the range of 4 to $24 \mu \mathrm{g} / \mathrm{ml}$ for BET and 3 to $18 \mu \mathrm{g} / \mathrm{ml}$ for PRO Prepared six times in this calibration range and absorbance determined at the respective wavelength for each drug alone. The results show good linearity between absorbance and concentration in the prescribed concentration range for both the drugs.

For simultaneous estimation of nitazoxanide and ofloxacin linearity perform as five different concentrations of standard mixtures prepared, $50 \%, 75 \%, 100 \%, 125 \%, 150 \%$ were injected, and chromatogram was recorded. The correlation coefficient was calculated and was observed to be greater than 0.99 for both the drugs which are within the limit.

The linearity of paracetamol was performed by preparing different concentrations $(6.25,12.5$, 25,50 , and $100 \mu \mathrm{g} / \mathrm{ml}$ ) from a stock solution of $10 \mathrm{mg} / \mathrm{ml}$. The solution of $20 \mu \mathrm{l}$ was injected into column three times. Linearity of paracetamol was found in the concentration range of $6.25-100 \mu \mathrm{g} / \mathrm{ml}$ with a correlation coefficient of 0.999 .

\section{Range}

It can be characterized as the interval amongst upper and lower quantities of analyte in the sample. Minimum of the specified range to be $80 \%$ to $120 \%$ of the test sample for the assay test $^{41}$.

\section{Ruggedness}

Ruggedness is the degree or measure of reproducibility under different situations such as in different laboratories, different analyst, different machines, environmental conditions, operators etc ${ }^{42}$. In the simultaneous estimation of nitazoxanide and ofloxacin, ruggedness was performed by different analyst and in different laboratories in different days to checks for any variation in the chromatography. The $\% \mathrm{RSD}$ for area and retention time was calculated for determination.

\section{Robustness}

It is characterized by the level of ability of an $\neg$ analytical technique, to stay similar by minute purposely change in the technique parameter. The different technique parameters which can be modified in high-performance liquid chromatography are $\mathrm{pH}$, drift rate, the temperature of the column and mobile phase composition $^{43}$.

In the simultaneous estimation of nitazoxanide and ofloxacin, the robustness of the proposed method verified by to perform analysis under variable flow rates. The flow rate as per the developed method is $1.5 \mathrm{ml} / \mathrm{min}$. Slight change in flow rate is $1.3 \mathrm{ml} / \mathrm{min}$ and $1.7 \mathrm{ml} / \mathrm{min}$ and chromatogram recorded. Due to a slight change in the flow rate of method shows good results and remain unaffected by that minute change. So, we can say that the method is robust.

For robustness of paracetamol, small modification in the flow rate, $\%$ of acetonitrile and $\mathrm{pH}$ of the mobile phase, the method remains 
unaffected and \% RSD value obtained are approximately near.

\section{Conclusion}

Validation is key element in assuming the quality of the product, Validation assures a great importance for quality assurance \& cost reduction. Validation produces product fit for intended use. Process validation is establishment and performance of activity required to obtain documented assurance that a manufacturing process or a part thereof - during routine use and Correct So that specified requirement on process variables and product properties are compiled with. Awareness about validation and it's process will help to ensure the reproducible quality products across the globe.

\section{References}

1. Khushboo DS et al., Sch. Acad. J. Pharm., 2014; 3(2):178-190.

2. Sree Ramamurthy, and Saravanakumar, M. Validation, The Eastern Pharmacist, 1997; $11: 476$.

3. Strictly As Per Syllabus Prescribed for M. Pharmacy (Pharmaceutical Quality Assurance), Semester-II by Pharmacy Council of India, New Delhi.

4. Chapman K. G: A History of Validation in the United States, Part I, Pharma Technology. November 1991; 39-98.

5. Nash R. A., Wachter A. H: In Pharmaceutical Process Validation. Third Edition; Revised and Expanded, Marcel Dekker Inc., New York, 1993.

6. Potdar MA; Pharmaceutical Quality Assurance. 2nd Edition, NiraliPrakashan, 2009: 8.6-8.20.

7. WHO Expert Committee on Specifications for Pharmaceutical Preparations. WHO technical report, series no. 863 - 34th report, Annex 6 - GMP: Guidelines on the validation of manufacturing processes: 4-7.

8. Patel Hiren Popatbhai et al. Process validation of Benazepril HCL 5mg tablet. International Research Journal of Pharmaceutical and applied sciences 2012; 2 (4): 1-16.
9. Venkata Raveendranath T. Process validation of Citalopram Hydro bromide tablets. International Journal of Research in Pharmaceutical and Biomedical Sciences 2010; 1 (2): 109-123.

10. Lavanya G, Sunil M, Eswarudu MM, Eswaraiah MC, Harisudha K, Spandana BN, et al. Analytical method validation: an updated review. Int $\mathrm{J}$ Pharm Sci Res 2013;4:1280.

11. Verma P, Madhav NS, KR Gupta V. A review article on pharmaceutical validation and process controls. Pharma Innovation 2012;1:51-60.

12. Bhardwaj SK, Dwivedi K, Agarwal DD. A review: HPLC method development and validation. Int $\mathrm{J}$ Anal Bioanal Chem 2015;5:76-1.

13. Goyal D, Maurya S, Verma C. Cleaning validation in the pharmaceutical industry-an overview. Pharma Tutor 2016;4:14-20.

14. Lodhi B, Padamwar P, Patel A. Cleaning validation for the pharmaceuticals, biopharmaceuticals, cosmetic and neutraceuticals industries. J Innov Pharm Biol Sci 2014;1:27-38.

15. Nash RA. Process Validation of a 17-Year retrospective study of solid dosage forms.DrugDevInd Pharm 1966; 22 (1): 25 34.

16. Jain, N.K., A text book of Pharmaceutical Product development, CBS publisher, 2005, 525-533.

17. Vandana B Patel. Studies in prospective process validation of Cimetidine tablet dosage form. International journal of Research in pharmaceutical and biomedical sciences 2011; 2(4): 1823-1836.

18. Venkata Raveendranath T. Process validation of Citalopram Hydro bromide tablets. International Journal of Research in Pharmaceutical and Biomedical Sciences 2010; 1 (2): 109-123.

19. Raveendranath T. Process validation of Pantoprazole tablets. The Pharma Innovation 2012; 1 (5): 53-73.

20. Nandha Kumar L. An overview of pharmaceutical validation- Quality 
Assurance view point. International journal of Research in pharmacy and chemistry 2011; 1(4): 1003-1014.

21. Rahul P. Process Validation of finasteride tablets. International journal of pharmaceutical chemical and biological sciences 2012; 2(1): 11-28.

22. Validation master plan installation and operation qualification non-sterile process validation cleaning validation. Pharmaceutical inspection convention pharmaceutical inspection cooperation scheme. www.picscheme.org.

23. ICH guidance for industry- Q8 Pharmaceutical development.

24. ICH guidance for industry - Q9 Quality Risk Management.

25. ICH guidance for industry - Q10 Pharmaceutical Quality Systems.

26. Notes for Guidance on Process Validation; CPMP/QWP/848/96, EMEA/ CVMP/598/99 September 2001.

27. Health Canada / Health Products and Food Branch Inspectorate Validation Guidelines for Pharmaceutical Dosage Forms (GUI 0029) / December, 2009.

28. Note for Guidance on Process Validation The Europe Agency fro Evaluation of Medicinal Products; CPMP/QWP/848/96; EMEA/CVMP/598/99;

29. Srivastava RK, Kumar SS. An updated review: analytical method validation. Eur J Pharm Med Res 2017;4:774-84.

30. Patel Paresh U. Development and validation of simultaneous equations method for estimation of betahistine dihydrochloride and prochlorperazine maleate in tablet dosage form. Inventi Rapid: Pharm Analysis and Quality Assurance; 2013.

31. Nirupa G, Tripathi UM. RP-HPLC analytical method development and validation for simultaneous estimation of two drugs nitazoxanide, ofloxacin and its pharmaceutical dosage forms. Int $\mathrm{J}$ ChemTech Res 2012;5:775-83.
32. Nayudu ST, Suresh PV. Bio-analytical method validation-a review. Int J Pharm Chem Res 2017;3:283-93.

33. Daksh S, Goyal A, Pandiya CK. Validation of analytical methods- strategy and significance. Int J Res Dev Pharm Life Sci 2015;4:1489-97.

34. Tijare LK, Rangari NT, Mahajan UN. A review on bioanalytical method development and validation. Asian J Pharm Clin Res 2016;9:6-10.

35. Devi TP, Setti A, Srikanth S, Nallapeta S, Pawar SC, Rao JV, et al. Method development and validation of paracetamol drug by RP-HPLC. J Med Allied Sci 2013;3:8.

36. Tiwari G, Tiwari R. Bioanalytical method validation: an updated review. Pharm Methods 2010;1:25-8.

37. Pasbola K, Chaudhary M. Updated review on analytical method development and validation by HPLC. World J Pharm Pharm Sci 2017;6:1612-30.

38. Geetha G, Raju KN, Kumar BV, Raja MG. Analytical method validation: an updated review. Int J Pharm Biol Sci 2012;1:64-1.

39. Shrivastava A, Gupta VB. Methods for the determination of limit of detection and limit of quantitation of the analytical methods. Chron Young Sci 2011;2:21.

40. Ravichandran V, Shalini S, Sundram KM, Harish R. Validation of analytical methodsstrategies and importance. Int $\mathrm{J}$ Pharm Pharm Sci 2010;2:18-2.

41. Shweta K, Anita S. A review on analytical method validation. Int $\mathrm{J}$ Pharm Res Rev 2016;5:30-6.

42. Boque R, Maroto A, Riu J, Rius FX. Validation of analytical methods. GrasasAceites2002;53:128-43.

43. PushpaLatha E, Sailaja B. Bioanalytical method development and validation by HPLC: a review. J Med Pharm Innov2014;1:1-9. 